\title{
High-Speed Rail Route and Regional Mobility with a Raster-Based Decision Support System: The Texas Urban Triangle Case
}

\author{
Hwan Yong Kim ${ }^{1}$, Douglas F. Wunneburger ${ }^{1}$, Michael Neuman ${ }^{2}$ \\ ${ }^{1}$ Department of Landscape Architecture and Urban Planning, Texas A\&M University, College Station, USA \\ ${ }^{2}$ Built Environment, The University of New South Wales, Sydney, Australia \\ Email: double.hs@gmail.com
}

Received October 30, 2013; revised November 30, 2013; accepted December 6, 2013

Copyright (C) 2013 Hwan Yong Kim et al. This is an open access article distributed under the Creative Commons Attribution License, which permits unrestricted use, distribution, and reproduction in any medium, provided the original work is properly cited.

\begin{abstract}
This study addresses sustainable transportation in the Texas Urban Triangle at the regional scale. Its aim is to determine the most suitable corridor for new transport infrastructure by employing a spatial decision support system proposed in this project. The system is being tested through its application to a prototype corridor parallel to Interstate 35 between San Antonio and Austin. The basic research questions asked are spatial in nature, so accordingly the geographic information system is the primary method of data analysis. The overall modeling approach is devoted to answering the following questions: What are the considerations to support sustainable growth? What scale or type of infrastructure is necessary? And how to adequately model the transportation corridors to meet the demands and to sustain the living environment at the same time?
\end{abstract}

Keywords: Spatial Decision Support System; Texas Urban Triangle; Geographic Information Systems; High Speed Rail

\section{Introduction}

The Texas Urban Triangle (TUT) is comprised of the metropolises of Dallas-Fort Worth, Houston, and San Antonio, and is distinguished among megalopolises because it is not linear but rather triangular. The axis from San Antonio to Dallas is on its way to becoming fully urbanized due to the proximity of the string of cities along Interstate 35 . In contrast, on Interstate 45 between Dallas and Houston, and on Interstate 10 between Houston and San Antonio, there are only small villages and towns along these arteries. Further, the urban development between its metropolises is not physically contiguous. The Texas Urban Triangle, with its massive number of inhabitants and area of more than 60,000 square miles, is the economic motor of Texas and hub of the national transportation network operating in a global economy. The Triangle is emerging as a new urban megaregion in its own right, competing with Los Angeles and New York, by virtue of its extensive internal connections and activities.

Accordingly, freight and passenger mobility within and among the Triangle's metro areas, as well as outward across the continent, are critical to economic and social development, and to the preservation of its natural assets. The initial analysis about the Triangle fringe revealed that over the next 20 years, population in the area will account for over 80 percent of the state's total [1]. The TUT is projected to account for $8,407,000$ of the state's $10,979,000$ new inhabitants, or 77 percent of all of Texas's growth [1]. The overall findings provide a baseline foundation for policy guidance to decision makers at all levels of government - especially state and federaland the private sector.

Given that transportation infrastructure shapes and supports growth, $\$ 58$ billion of the $\$ 72$ billion identified in Texas transportation infrastructure needed over the next 25 years is in the Texas Urban Triangle [2]. Existing highway-dominated surface transport systems are exceeding design capacity and are increasingly costly to expand and maintain. Accordingly, there is an urgent need for policy and investment decisions that are based on a new and wider set of criteria that account for new conditions and considerations. A new form of decision 
making based on emerging realities could pave the way for a wider range of options for transportation that are sustainable.

This study addresses sustainable transportation in the Texas Urban Triangle at the regional scale. Its aim is to determine the most suitable corridor for new transport infrastructure by employing a spatial decision support system (SDSS) proposed in this project. The SDSS is being tested through its application to a prototype corridor parallel to Interstate 35 between San Antonio and Austin. The basic research questions asked are spatial in nature, so accordingly the geographic information systems (GIS) are the primary method of data analysis. The overall modeling approach is devoted to answering the following questions: What are the considerations to support sustainable growth? What scale or type of infrastructure is necessary? And how to adequately model the transportation corridors to meet the demands and to sustain the living environment at the same time?

\section{Review of the Existing Transportation Decision Support Systems}

There are a wide range of decision support systems for transportation investments. Increasing relationship between transportation and land use developed relevant software packages. As a result, a large amount of decision support models are implemented by different organizations. In this section, a few different existing decision support systems are reviewed.

\subsection{TransDec2.0}

Developed by the Texas Transportation Institute (TTI) under National Cooperative Highway Research Program (NCHRP), TransDec is a multimodal investment model that takes into account many factors not easily measured in traditional benefit-cost assessments of project desirability, such as air quality considerations, gross mobility impacts, community livability factors, and aesthetic considerations [3]. TransDec uses multi-criteria utility analysis methods to assess tradeoffs between transportation modes, planning methods, and priorities set by project evaluators.

\subsection{Development of Intercity Passenger Network in Texas (TxDOT Project 0-5930)}

This project developed a state-wide network to move people between urban regions by either passenger rail or intercity bus services. For each intercity corridor, a set of criteria was developed to compare the suitability of each corridor against the others. Criteria utilized for this project include the population along each corridor, population density, projected population growth, total employees, number of public or private universities, air passen- ger travel between corridor airports, vehicular traffic, percent trucks, and average number of corridor flights per day [4]. The outcome of this evaluation will be the recommendation of which corridors are most likely to support an intercity transit system and whether bus or rail is most suitable.

\subsection{MicroBENCOST Model}

The MicroBENCOST software was developed by TTI researchers in the mid-1990s [5]. It provides a planninglevel economic analysis tool that can be used to analyze a variety of transportation projects. MicroBENCOST is designed to analyze different types of highway corridors. Benefits are calculated for existing and induced traffic, as well as for diverted traffic. Eight different traffic allocation options exist, depending on the traffic year and the nature of the marginal user costs considered.

\subsection{UPlan}

This is a GIS-based urban growth model that runs in the Windows version of ArcView. The model was designed by the research team to rely on a minimum amount of data, but it allocates urban growth in several land use types for small (parcel-sized) grid cells [6]. It is a scenario-testing model and rule based; i.e., it is not strictly calibrated on historical data and uses no choice or other statistical models. The result can be applied to various urban impact models to forecast soil erosion, local service costs, and other impacts.

\subsection{The "ALLOT" Model}

This model is an early prototype of the SDSS developed in 1992 in an attempt to provide governmental jurisdictions and private landowners with more economically efficient and environmentally sound land use and development patterns than usually occur [7]. It employed a GIS land suitability analysis model and multi-attribute value method that helped to determine the location of lands suitable for different land uses.

As can be seen, each model has its own emphasis and aims. But the problem stems from their data-intensive nature and the limited windows for user involvements. In addition, it is also unclear that most of the systems do not specify the relationship between transportation corridors and possible environmental changes. They rather concentrate heavily on the economic side. The proposed SDSS differs from the existing decision systems in that it focuses on selected strategic driving forces of growth of the region as a functional unit-transport infrastructure, available land, economic activity, water, energy, and so on-and then identifies corresponding measures of sustainability for key transportation systems and corridors within the TUT. For example, development patterns 
driven by transportation infrastructure in turn create impacts on surface and groundwater resources in terms of water quantity and quality. Conversely, consideration about water availability and waste assimilative capacity can be used as a driver of infrastructure planning decisions to achieve greater long-term sustainability.

The SDSS provides a composite foundation for policy analysis and support policy making for a comprehensive sustainable regionalism. The SDSS can be modified by users to support location decisions regarding local and state transportation corridors, in addition to metropolitanand regional-scale corridors. Moreover, it can be used to evaluate other types of infrastructure corridors that can be placed in shared rights of way within or alongside transportation corridors. Unlike the previously reviewed models, the SDSS is multi-scalar in addition to multiattribute, and represents an advance in decision support system model development.

The proposed SDSS compiles decision criteria into a land suitability analysis model [8], employing GIS to map strategic social, economic, and environmental characteristics, and overlay them to assess which locations are most and least suitable for regional transportation networks and urban-scale growth. The vastly changed transportation investment decision panorama in Texas and the United States implies a new type of decisionmaking that considers more than just capital costs and environmental constraints. It needs to consider the economic, demographic, social, ecological, infrastructural, and fiscal parameters influencing decisions.

\section{Pre-Modeling}

\subsection{Identify Factors}

"Factors" in the SDSS refers to the individual criteria used in the model to assess the most suitable location for placing transportation corridors on the landscape. Utilizing the Delphi panel discussion technique, a set of experts panel was formed and initially selected 83 factors that could be included in the SDSS criteria. They are organized into seven categories: culture; demographics; engineering; environment; hazard; natural resources; and lands. After thorough deliberation, 38 factors appropriate for a transportation corridor were identified, and this study adopts seven of them to examine the modeling process.

Table 1 presents the list of factor selection. Type of roads, Floodplain, and Surface waters are selected to minimize the probability in constructing additional structures to cross any highways or rivers. Slope, Geology, and Soil types are chosen to represent the construction suitability (i.e. earthworks or foundation enhancements). Finally, Population density is used to minimize any conflicts in relocating people and goods.
Table 1. Selected factors.

\begin{tabular}{ccc}
\hline No. & Categories & Indicators \\
\hline 1 & Engineering & Roadway right of way (type of roads) \\
2 & & Slope/maximum grade \\
3 & & Surface waters (hydrologic units) \\
4 & Geology/faults \\
5 & Sonvironmental & Floodplain \\
6 & & Population density \\
7 & Demographics & \\
\hline
\end{tabular}

In some cases, a factor is simple binary, such as present or not present, or should or should not exist within the transportation corridor. In other cases the factors are quantitative, and in still other cases the factors are qualitative. Some factors mark a gradient or a range within which there are acceptable (suitable) or non-acceptable (non-suitable) values. Since this is closer to a pilot test, we decided to implement these factors into the limited geographic area, and test the model operation as intended. Accordingly, six counties: Travis, Hays, Caldwell, Comal, Guadalupe, and Bexar, were selected to represent the locations for a possible route between Austin and San Antonio.

\subsection{Data Sources and Preparation}

Based on factor identification, corresponding datasets for each factor are gathered. In specific, population datasets are collected from the US Census Bureau. The Texas Natural Resource Information System (TNRIS) website provides comprehensive datasets for the State of Texas, and the author obtained hydrology and transportation data. Transportation datasets are obtained from the Texas Department of Transportation (TXDOT). The Federal Emergency Management Acts (FEMA) provides datasets about the floodplains. Vertical slope or the maximum grade is calculated using the Digital Elevation Model (DEM) easily accessible at the US Geological Survey (USGS). The USGS website has a massive amount of geographical datasets, from which geology data are also acquired. Finally, soil data are discovered using the National Resources Conservation Services (NRCS) website. Some other governmental agencies such as the Capital Area Council of Governments (CAPCOG) or the Alamo Area of Council of Government (AACOG) are also helpful sources for collecting datasets.

Most of the acquired datasets are in feature classes, meaning that they are in vector formats. In order to conduct the proposed SDSS with the GIS, all the datasets need to be converted into raster formats. A raster consists of a matrix of cells (pixels) organized into rows and columns, where each cell contains a value representing 
information, such as temperature or vegetation covers [9]. By implementing raster datasets, users can operate pixel values and GIS provides several functions to analyze or recreate information by manipulating pixel values. This information-processing step is the key to setting up the optimal path in route modeling.

In addition, raster format has to be in the same pixel size. In this case, a $30 \mathrm{M} \times 30 \mathrm{M}$ cell size was implemented. The main reason is to minimize the information loss during the data processing period. When users use many datasets, the best way to handle the information loss is aligning to the minimum cell size across the datasets. In this case, all the vector datasets are converted into $30 \mathrm{M} \times 30 \mathrm{M}$ grid because the initial Digital Elevation Model (DEM) used to calculate the vertical slope was in $30 \mathrm{M}$ format, and the previous studies indicate that the typical right-of-way of a high-speed rail is $30 \mathrm{M}$ for one way [10].

\subsection{Classification}

Factor classification refers to assigning the values, on a scale of 1 to 5 , to each pixel of the factors (decision criteria) in the SDSS. A value of five is the highest score in a factor and reflects a negative value for locating a transportation corridor in that places - the least suitable location. A value of 1 is the lowest score in a factor and reflects a positive value for locating a transportation corridor - the most suitable location.

Some factors such as roads and hydrology were classified into binary (present or not present type). For example, the author concluded that constructing a high-speed rail over an interstate highway requires a complex decision-making process as well as higher costs. In this sense, having a rail route over an interstate highway receives a higher score (less suitability) than doing so over a local street. Similar logic applies to the hydrology. The major stream category receives a higher score (less suitability) than the others. This reclassification process involves intensive inputs from both professionals and previous studies. Based on relevant literature reviews, each factor is scored. Some other factors without strong theoretical backgrounds utilized experts' opinions instead.

This classification process is important because it proposes a window to reflect various inputs from the general public. If relevant stakeholders could involve in this classification process, the outcome would become more participatory. For now, the model utilizes inputs only from the experts. But in future, developing a method that will incorporate public participation in this reclassification as well as some other processes will greatly enhance the reliability of the outcome. This possibility distinguishes the proposed SDSS with other decision support systems, and opens up for more participatory GIS.

\subsection{Factor Weights}

Importance of factors to the route implies a few different meanings and in this case, the cost side of HSR is more considered than the others features such as environment or operation as this is closer to model-testing. Consequently, a weight matrix is created to define the relationship between the factors, and named as the factor weights matrix. Factor weights refer to the relative value of factors compared to each other. Thus, the most important factor to consider for locating a HSR in the Texas Urban Triangle is weighted as the highest, the second most important factor is the next, and so on, until the least important factor.

In order to determine appropriate factor weights, the Analytic Hierarchy Process (AHP) is applied. Developed by Thomas Saaty in the 1970s, the AHP helps organize and analyze complex decisions. It is based on the welldefined mathematical structure of consistent matrices and their associated right eigenvector's ability to generate true or approximate weights [11]. The AHP is a widely accepted decision-making strategy, especially when dealing with various datasets with multiple criteria. In this case, the relationship between seven selected factors is tested with the AHP. Table 2 illustrates the AHP result. Eigen vectors show the factor weight for each factor and are standardized values.

Weights are allocated in a subsequent order: Population Density $\geq$ Slope $>$ Roads $>$ Geology $>$ Soil Type $>$ Hydrology = Floodplain. This order is based on the degrees of anticipated difficulties in railroad construction. Relocating people from one place to another not only requires cost, but also needs extensive amount of conflict resolution. Constructing a railroad on the ground with more than 3.5\% slope is not impossible, but requires excessive costs. A Californian study limits vertical slope to $3.5 \%$ as it is in the exceptional grades range [10]. Therefore, slope is considered as important as relocating people and goods, and set to the same weight as density. Roads are one of the significant obstacles in railroad construction, and valued as the third highest. The most expected problem in Roads factor is crossing. If a route encounters a freeway in the middle of its operation, it should either detour or find a new way to go across.

In any case, both the economic and social burdens will substantially increase. Geology and Soil Types relate to two particular aspects: construction and operation. Having solid grounds enables safe construction and stable vehicle operation. The last two are Hydrology and Floodplain. Since this study is more concentrated on construction suitability, the author valued these two factors relatively lower than the other factors. However, if environmental impact is more of a concern, these two can be valued higher, and this is one of distinctions that the proposed SDSS differs from the reviewed studies previ- 
Table 2. AHP result.

\begin{tabular}{cccccccccc}
\hline & Density & Slope & Roads & Hydrology & Floodplain & Geology & Soils & Eigen vector & Cumulative \% \\
\hline Density & 0.33 & 0.40 & 0.29 & 0.24 & 0.20 & 0.20 & 0.23 & $\mathbf{0 . 2 7}$ & $29.0 \%$ \\
Slope & 0.17 & 0.09 & 0.14 & 0.24 & 0.18 & 0.23 & 0.29 & $\mathbf{0 . 1 8}$ & $19.0 \%$ \\
Roads & 0.22 & 0.27 & 0.43 & 0.24 & 0.16 & 0.23 & 0.23 & $\mathbf{0 . 2 7}$ & $27.0 \%$ \\
Hydrology & 0.07 & 0.05 & 0.03 & 0.05 & 0.08 & 0.02 & 0.02 & $\mathbf{0 . 0 4}$ & $5.0 \%$ \\
Floodplain & 0.07 & 0.07 & 0.03 & 0.02 & 0.04 & 0.02 & 0.02 & $\mathbf{0 . 0 4}$ & $4.0 \%$ \\
Geology & 0.11 & 0.08 & 0.04 & 0.17 & 0.16 & 0.07 & 0.09 & $\mathbf{0 . 1 0}$ & $9.0 \%$ \\
Soils & 0.08 & 0.07 & 0.03 & 0.17 & 0.14 & 0.04 & 0.06 & $\mathbf{0 . 0 8}$ & $8.0 \%$ \\
SUM & 1.04 & 1.02 & 0.99 & 1.15 & 0.96 & 0.80 & 0.94 & $\mathbf{1 . 0 0}$ & $100 \%$ \\
\hline
\end{tabular}

$\lambda_{\max }=7.7070$, consistency index $(\mathrm{CI})=0.1178$, random index $(\mathrm{RI})=1.32(\mathrm{n}=7)$ consistency ratio $(\mathrm{CR})=0.0893<0.1$.

ously. Users decide to which regard the factors to be valued, or to develop a possible scenario with the given weights. This is important because implementation of user judgment in weigh allocation opens up for another possibility for participatory GIS.

One way to determine the reliability of the created weight matrix is using the Consistency Ratio (CR). CR indicates the consistency of weight matrix if the AHP process is done repeatedly [12]. In our case, the CR came out to be less than $10 \%(8.93 \%)$, and this implies that the created weight matrix has an error variance less than 10 percent. In most studies applying AHP, a CR value less than 10 percent is considered a reliable measure [13].

The relationship between the factors and factor weights are a crucial part of the entire SDSS process. The way the factor weights are determined could give different result to the final routes. How participants set up factor weight creates different route options and gives different implications in terms of transportation investment. Comparing diverse results with participants' own goals is one of useful tactics in decision-making process. In this case, setting up the relevant importance of each factor was done with in-depth discussions between the author and factor experts. Similar to the internal classification process, it is possible for the participants to elaborate the weighting process based on their own goals and objectives, and this is another possibility of incorporating participatory GIS environment to the proposed SDSS.

\section{GIS-Modeling}

Based on the previous steps, raster-based GIS modeling is utilized. The raster-based modeling is powerful when new information is in demand based on existing conditions. By converting all datasets into raster formats, we obtain new information in a pixel level. For example, if we decide that population density less than 10 (10 people/acre) is equal to a suitability score of 2 , then all the pixels with population density less than 10 are converted into a score of 2 . This process involves two particular steps: creating cost surfaces with the given weights of factors and extracting the shortest path on a cell-by-cell basis.

\subsection{Cost Surfaces}

Using factor weights, an equation is derived to create a unified cost surface. By adding up the factors multiplied with their relevant weights in AHP result, a single map implying suitability scores is created. As mentioned earlier, all factor datasets are prepared in a $30 \mathrm{M} \times 30 \mathrm{M}$ gird format. In other words, all the pixels in a factor dataset have a value of 1 through 5 as set in the classification step. Multiplying weights to factors implies multiplying factor weights to the entire pixel values in dataset. Therefore, the cost surface is also in a grid format with $30 \mathrm{M} \times 30 \mathrm{M}$ pixels. The map algebra can be written as follows:

$$
\text { Final Suitability Surface }=\sum_{k} w_{k} x_{i j}
$$

(where $w_{k}$ : external weight for factor $k, x_{i j}$ : value of a grid cell $i j$ in factor $k$.)

Figure 1 represents the results of using the equation with the factors. By adding up all the factors, pixels in the cost surface acquire the suitability scores. This score is standardized to fit into a 1-to-5 scale, meaning that a pixel value closer to 5 indicates negative suitability for a HSR route. Unlike the convention, a reversed scale is used because finding the shortest path in GIS is based on the least possible pixel scores between the departure and destination. In other words, the optimal route will be drawn by connecting all the least possible scores between the origin and destination. The lower the suitability scores, the better fit for the shortest path.

Due to the reason that the author set the highest weights to population density, the suitability scores on the major population centers came out the be the highest-low suitability. City boundaries of Austin, San Antonio, San Marcos, and New Braunfels are the places showing the lowest suitability for the HSR route. Further, 
geology, soil type, and slope factors made low suitability on the east part of the study area.

\subsection{Least Path Analysis}

The next step is to perform the shortest path analysis. GIS finds the pixels with the least possible scores between the two designated points. After setting up the locations of origin and destination points, combined functions in GIS seek for the least possible scores around each proceeding pixel. The least possible scores are constantly identified and connected until the path reaches its final destination.

For research convenience, the departure and destination points were set to two cities' major airports. Station location involves a different set of decision-making procedure and could become a highly political agenda. Therefore, the author decided to drop station location decision at this moment, and arbitrary set to two major airports: Austin-Bergstrom Airport and San Antonio International Airport. Whether the location is set to airports or different geographic locations, the route modeling process does not change and the result will be drawn accordingly.

Figure 2 represents the suitability map with the shortest path extracted. This path is drawn based on each pixel's suitability score. As can be seen, the route intentionally avoids major population centers. In addition, because most of low suitable areas are located on the southeast side of the study boundary, the route rather indicates as a straight line. Because this line is basically a vector line connected with pixel by pixel, however, the shape is not a curve and thus, requires a post-smoothing process.

\subsection{Smoothing Process}

The shortest path analysis finds the most suitable route by searching each pixel's score in the cost surface. The result is in a vector line connecting the most suitable 30 $\mathrm{M} \times 30 \mathrm{M}$ pixel. However, post-processing is required to smooth the line to the parameters required for construction. Construction parameters applied to high-speed rail require turns must be limited to curves defined by a minimum of 4.5-mile radius [14]. This restriction is hard to apply in the proposed model because this is closer to a master plan stage, not a specific engineering process.

Using GIS, however, users are able to comply with such restrictions in a few different ways. For example, the moving window analysis will be a good starting point to analyze the radius of a route, but may require significant time and resources to analyze all the pixels within that regard. In this case, the study used "Smooth" function provided in the Advanced Editing toolbox. The extent of smoothing is set by indicating the maximum al-

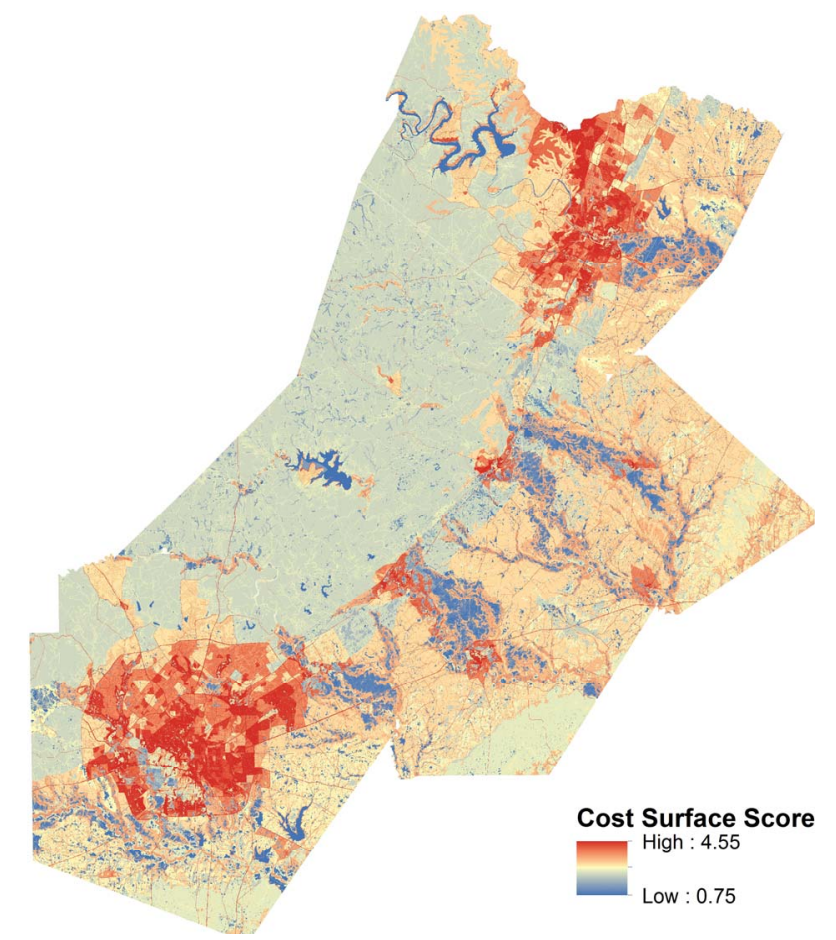

Figure 1. Final cost surface with AHP result.

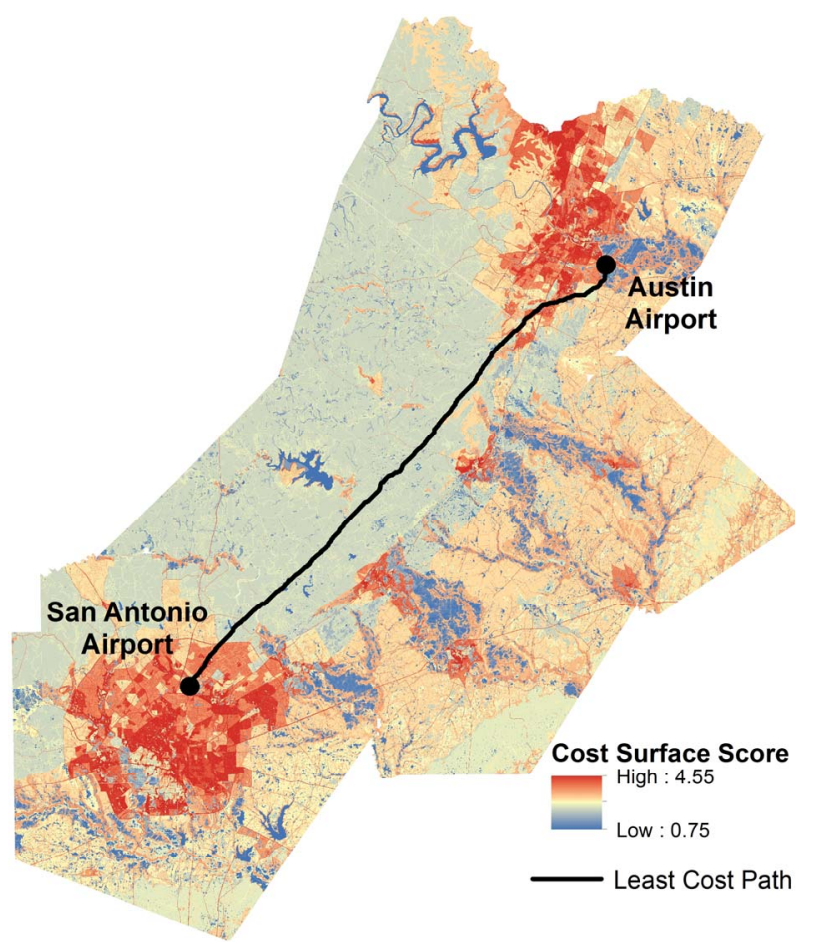

Figure 2. Least cost path with the cost surface.

lowable offset, limiting the maximum distance the output geometry (curves) can be from the input geometry (vertices) [15]. Smoothening process may not strictly enforce the route with the 4.5 -mile radius specification. But it gives us a brief idea about how the route will look in 
reality with the radius restriction.

\section{Result}

During the previous sections, an optimal HSR route was extracted based on the initial judgment criteria and input factors. The route is designed to intentionally avoid population center, which is the study's purpose at the beginning, and find out the least conflict in construction suitability, which is determined by geology, soil type, and slope factors. In this case, the subsequent step would be understanding the result. There are a few different ways to interpret the route, and in this study we focus on the total length, travel time, and expected construction estimates. As this study is prototype modeling test and a part of a longitudinal research project, more elaboration about the final route will be followed in the later studies.

In 2011 summer, the TUT team at Texas A\&M University had an opportunity to have a meeting with a Korean engineering firm. The meeting was about technology consultation and transfer. During the meeting, researchers had a chance to receive a detailed estimate of the Korean HSR construction costs. As the State of Texas has not decided which HSR vehicles to use, this study implements the Korea HSR specification to estimate anticipated costs for its construction.

Table 3 represents the total length of the final route with the $60 \mathrm{M}$ of right-of-way (ROW). A $60 \mathrm{M}$ ROW is used because it is for a two-way HSR track [16]. In addition, the Korean HSR is operating with the speed of 200 mile/hour. Although the average speed across the entire route will slower than $200 \mathrm{MPH}$, the constant speed was assumed because the total length is relatively short and less number of stations between the two points are expected.

As can be seen, the extracted route consumes 1715 acres of lands for its rail tracks and takes about 22 minutes to reach the San-Antonio International Airport from the Austin-Bergstrom International Airport with the speed of 200 mile per hour. In addition, the estimated total construction costs using the Korean HSR specifications came out as $\$ 55.6$ Million, which is approximately $\$ 0.77$ million for one-mile of railroad construction.

\section{Conclusion}

The proposed SDSS is robust, meaning that it is supported by valid theory, developed by using a sound methodology, and based on reliable and accurate data. This robust quality, coupled with the wide range of factors in the SDSS model, enables it to be adapted to a wide range of geographic and technological circumstances beyond Texas and its Urban Triangle, depending on the intended use. A wide range of geographic conditions means two things: places throughout the United States and the world,
Table 3. Cost estimate and travel time.

\begin{tabular}{cc}
\hline & Route information \\
\hline Total length & 71.9 miles $(=115,828$ meters $)$ \\
Travel time @ 200 MPH & 0.36 hours $(=22$ minutes $)$ \\
Track area w/60 M ROW & 1715 acres $\left(=6,940,888 \mathrm{~m}^{2}\right)$ \\
Construction cost estimate & $\$ 55.6$ million \\
\hline
\end{tabular}

not just Texas; and a range of scales from the municipality to the multi-state region. The wide range of technological circumstances means any type of ground transportation technology or mode, whether rail, road, or multimodal.

Furthermore, the adaptability and flexibility of the model is afforded by the ability of any user to tailor the input factors to suit the scale and territory to which it is applied. For example, if a region is heavily forested and topographically rugged, those two environmental characteristics can be bolstered with additional factors, and those two factors can be adjusted to suit the specific local conditions. Moreover, end users can organize both the internal classifications within each factor and the external weights among the factors as compared to the other factors selected. The capacity of this decision support system can be expanded to make more complex decisions by incorporating other, diverse characteristics and using them as additional inputs into the SDSS. By doing so, not only does new transportation infrastructure mean an opportunity for new economic possibilities and new urban development, but its right of way can also be located to support sustainable development for the future.

The study analyzed a possible HSR route emphasizing the construction aspect of HSR. The result indicates that the initial investment of $\$ 55.6$ million would be required to build a HSR route in between San Antonio and Austin. In addition, the optimized rail track will consume around 1700 acres of lands with the two-way ROW, and will reduce the travel time to 22 minutes, compared to that which takes about 1.5 hours with an automobile. The suggested modeling process and interpretation of the result imply a possible way to conceptualize the route information with the given criteria. More importantly, this study provides a foundation that will be implemented to further studies regarding participatory GIS in decision-making environment.

There are some margins to be developed in the SDSS and the Texas Urban Triangle at large. The proposed SDSS should be constantly tested and developed for the rational investment decision in the Texas Urban Triangle area. The SDSS would be applied to actual decision making for transportation corridors in the Texas Urban Triangle in concert with key regional transportation entities, including but not limited to the four principal metropoli- 
tan planning organizations and councils of governments in the Triangle, as well as the Texas Department of Transportation.

In addition, future users should modify the SDSS to address perceptions held by stakeholders. For example, additional factors may be beneficial for analyzing and mitigating adverse impacts of large ownerships by fragmentation. To comprehend the limitation, more studies are under review to improve the overall modeling process.

\section{Acknowledgements}

This study is part of a longitudinal study about Texas Urban Triangle. The author gratefully acknowledges the University Transportation Center for Mobility at the Texas Transportation Institute for support. The author would also like to thank POSCO Engineering, Inc. for their provision of the Korean high-speed rail information.

\section{REFERENCES}

[1] M. Neuman and E. Bright, "Texas Urban Triangle: Framework for Future Growth," Southwest Region University Transportation Center, College Station, 2008.

[2] Governor's Business Council Transportation Task Force, "Shaping the Competitive Advantage of Texas Metropolitan Regions," Governor's Business Council, Austin, 2006.

[3] S. Roop, "TransDec 2.0: Project Planning Decisions Made Easy. Texas Transportation Researcher," Texas Transportation Institute, College Station, 2003.

[4] C. A. Morgan, B. R. Sperry, J. E. Warner, A. A. Protopapas, J. D. Borowiec, L. L. Higgins and T. B. Carlson, "Potential Development of an Intercity Passenger Transit System in Texas," Texas Transportation Institute, College Station, 2010.

[5] British Columbia Ministry of Transportation, "MicroBENCOST Guidebook: Guidelines for the Benefit Cost Analysis of Highway Improvement Projects in British Columbia," British Columbia Ministry of Transportation, British Columbia, 2005.
[6] R. A. Johnston, D. R. Shabazian and S. Gao, "UPlan: A Versatile Urban Growth Model for Transportation Planning," Transportation Research Record 1831 (2003), 2007, pp. 202-209.

[7] E. M. Bright, “The 'Allot' Model: A PC-Based Approach to Siting and Planning," Computers, Environment and Urban Systems, Vol. 16, No. 5, 1992, pp. 435-451. http://dx.doi.org/10.1016/0198-9715(92)90004-B

[8] I. L. McHarg, "Design with Nature," J. Wiley, New York, 1994.

[9] C. P. Lo and A. K. W. Yeung, "Concepts and Techniques of Geographic Information Systems," Pearson Prentice Hall, Upper Saddle River, 2006.

[10] Parsons Brinckerhoff, "Technical Memorandum: Alignment Design Standards for High-Speed Train Operation TM 2.1.2. California High-Speed Train Project," California High-Speed Rail Authority, Sacramento, 2009.

[11] T. L. Saaty, "The Analytic Hierarchy Process: Planning, Priority Setting, Resource Allocation," RWS Publications, Pittsburgh, 1990.

[12] R. Ramanathan, "A Note on the Use of the Analytic Hierarchy Process for Environmental Impact Assessment," Journal of Environmental Management, Vol. 63, No. 1, 2001, pp. 27-35. http://dx.doi.org/10.1006/jema.2001.0455

[13] E. H. Forman, "Random Indices for Incomplete Pairwise Comparison Matrices," European Journal of Operational Research, Vol. 48, No. 1, 1990, pp. 153-155. http://dx.doi.org/10.1016/0377-2217(90)90072-J

[14] The European Parliament and of the Council, "Directive on the Interoperability of the Rail System within the Community," Official Journal of the European Union, Brussels, 2008, pp. 1-191.

[15] ArcGIS Resource Center, "Smooth Line (Cartography)," 2010.

http://resources.arcgis.com/en/help/main/10.1/index.html \#//007000000012000000

[16] California High-Speed Rail Authority, "Final Program Environmental Impact Report/Environmental Impact Statement (EIR/EIS) for the Proposed California High-Speed Train System," USDOT Federal Railroad Administration, Sacramento, 2005, p. 75. 